\title{
Perceptual saliency of points along the contour of everyday objects: A large-scale study
}

\author{
Joeri De Winter and Johan Wagemans \\ University of Leuven, Leuven, Belgium
}

\begin{abstract}
The aim of this large-scale study was to find out which points along the contour of a shape are most salient and why. Many subjects $(N=161)$ were asked to mark salient points on contour stimuli, derived from a large set of line drawings of everyday objects $(N=260)$. The database of more than 200,000 marked points was analyzed extensively to test the hypothesis, first formulated by Attneave (1954), that curvature extrema are most salient. This hypothesis was confirmed by the data: Highly salient points are usually very close to strong curvature extrema (positive maxima and negative minima). However, perceptual saliency of points along the contour is determined by more factors than just local absolute curvature. This was confirmed by an extensive correlational analysis of perceptual saliency in relation to ten different stimulus factors. A point is more salient when the two line segments connecting it with its two neighboring salient points make a sharp turning angle and when the 2-D part defined by the triplet of salient points is less compact and sticks out more.
\end{abstract}

It has been a well-known fact since the early days of vision science (e.g., Alhazen, ca. A.D. 1030; see Sabra, 1989) that not all points along an object's boundary contour are equally informative about that object's shape. Attneave (1954) was probably the first to explicitly formulate the hypothesis that curvature extrema (i.e., points along the contour where curvature reaches a local maximum) are most informative about shape. He used two demonstrations to support this hypothesis. In one demonstration, he asked subjects to mark salient points along the contour of a random shape and he showed that the frequency plots were centered on the curvature extrema (see Figure 1A). In a second demonstration that has become known as Attneave's sleeping cat he created a version of a line drawing of his sleeping cat by connecting the curvature extrema by straight lines and he showed that this straight-line version was still easy to recognize.

Kennedy and Domander (1985), however, questioned Attneave's (1954) hypothesis that points of maximum curvature are most informative for shape recognition. In three experiments, with a small number of fragmented contour stimuli depicting manmade objects, they showed that identification of these stimuli was better when fragments were placed midway between extrema, and best when fragments were placed midway between midpoints and extrema. Kennedy and Domander concluded that the shapes of objects are best represented by samples of the contour that are selected to be evenly distributed, even if this means eliminating all of the points where curvature changes direction maximally (i.e., curvature extrema).

In a more recent study, with 12 silhouette stimuli derived from the shadows cast by sweet potatoes, Norman,
Phillips, and Ross (2001) attempted to replicate Attneave's (1954) first demonstration more closely. Their results supported Attneave's hypothesis. More specifically, 12 subjects were asked to "copy" the silhouettes by positioning 10 points until the dotted contour version resembled the original silhouette version as closely as possible. The frequency plots (see Figure 1B) looked very similar to the one from Attneave's study (see Figure 1A). In addition, by doing a curvature analysis, they were able to derive the strongest curvature extrema, positive maxima, as well as negative minima (indicated by closed and open circles, respectively; see Figure 1C). By superimposing the points selected by at least half of the observers (indicated by arrows; see also Figure 1C), they then showed that the most informative points were almost always very close to the curvature extrema.

Norman et al. (2001) attributed the discrepancy between their results and those of Kennedy and Domander (1985) to stimulus and task differences. Kennedy and Domander had used fragmented contour stimuli depicting manmade objects (a window, a box, a stove, an electric clothes dryer, etc.), consisting mainly of rectangular surfaces bordered by straight edges; the only "curved" parts were sharp corners where the straight edges intersected. Norman et al., in contrast, used natural solid objects with convex and concave surface patches, projecting to smoothly varying convex and concave contour curvature (with few straightline segments and few sharp corners). Moreover, subjects in Kennedy and Domander's study had to identify the fragmented contour pictures, whereas Norman et al.'s observers were asked to indicate important points while they inspected the complete silhouette stimuli. 


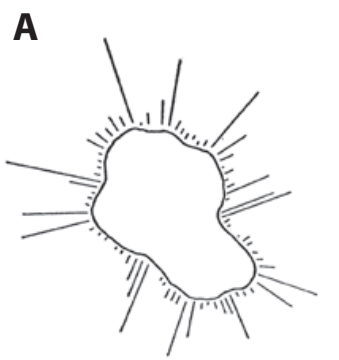

B

C

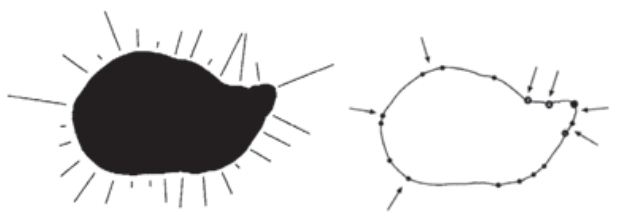

\begin{abstract}
Figure 1. (A) Attneave (1954) demonstrated the importance of curvature extrema by showing that people mark them as more salient points along the contour of a random shape (higher frequency is represented by a longer line). This demonstration received further empirical support from a study by Norman et al. (2001) with 12 observers and 12 silhouettes (cast shadows from sweet potatoes). (B) One example of a stimulus and a frequency plot as in Attneave's demonstration (No. 1 from Figure 2 in Norman et al., 2001). (C) Arrows indicate locations along the contour marked by half or more of the observers, whereas circles indicate curvature extrema (closed ones indicate positive maxima or $M+$, open ones indicate negative minima or $m-$ ); the radius of the circle indicates the magnitude of the absolute curvature (No. 1 from Figure 5 in Norman et al., 2001). It is clear that the most salient points are situated at or near extrema, although not all extrema are equally salient.
\end{abstract}

Attneave's (1954) hypothesis has not only triggered empirical research; it has also inspired more theoretical work in at least two different directions. First, Resnikoff (1989) has formalized the notion of information using classic measures from information theory, and he has provided mathematical proof of the information concentration in curvature extrema. More recently, Feldman and Singh (2005) proposed an alternative quantification scheme. They extended Attneave's original idea by showing that the same logic implies that negative curvature extrema (minima) must be more informative than positive curvature extrema (maxima) because all natural objects are more convex than concave; closed contours must, therefore, always turn inward after turning away from the object center. Second, using concepts and formalizations from projective and differential geometry, Koenderink and van Doorn (1976; Koenderink, 1984) have shown that contour fragments of positive curvature (or convexities) correspond to regions of positive Gaussian curvature on the 3-D surface (i.e., bump-like regions), whereas contour fragments of negative curvature (or concavities) correspond to regions of negative Gaussian curvature on the 3-D surface (i.e., saddle-like regions). Inflections (points where curvature changes sign and goes through zero locally) might then be informative points too, because they correspond to parabolic lines on the 3-D surface, separating convex and concave surface regions (Koenderink \& van Doorn, 1982; Van Gool, Moons, Pauwels, \& Wagemans, 1994).

The present study had two major goals. First, we wanted to test Attneave's (1954) hypothesis about the importance of curvature extrema for shape perception, using a much larger set of stimuli and observers than in previous studies. In another large-scale study, we created silhouette and outline versions of the famous Snodgrass and Vanderwart (1980) set of line drawings of everyday objects, and we obtained identification norms for them (Wagemans et al.,
2007; see also the Appendix in Wagemans, Notebaert, \& Boucart, 1998). Because we have continuous curvature values along the contours of these outlines, we can now address several hypotheses about the role of curvature singularities in shape perception in a thorough and systematic way. One step in this research program (for an overview, see De Winter \& Wagemans, 2004), consists in asking whether subjects mark curvature extrema (or points in their close neighborhood) as salient points. Our stimulus set is probably ideal to address the issue of stimulus variability, which appeared to be at stake when the results of Kennedy and Domander (1985) were compared with those of Norman et al. (2001), because Snodgrass and Vanderwart's set of line drawings was taken to be representative of all kinds of everyday objects, biological as well as manmade.

Second, we wanted to provide solid empirical data about perceptual saliency of contour points on outlines of everyday objects as a necessary stepping stone to performing experiments on identification of straight-line versions (De Winter \& Wagemans, in press) and fragmented versions (Panis, De Winter, Vandekerckhove, \& Wagemans, in press), using empirically validated points to connect by straight-line segments, or to position contour fragments. In the present study, we used purely subjective judgments (i.e., we asked subjects to mark points that they considered to be salient), but these additional identification studies will allow us to corroborate our findings with more objective performance data (see General Discussion). When we use the term "perceptual saliency," with reference to our results, we mean "perceptual saliency as indicated by our subjects when they marked points along the contour."

The article consists of three major parts. First, we describe the methods of data acquisition and analysis and we report some descriptive statistics. Second, we present a thorough analysis of perceptual saliency, using several tools to obtain meaningful aggregated values from the huge 
database of empirically marked points. We also relate the location of the selected points to the location of curvature singularities. Third, we report results from an exploratory a posteriori analysis, in which we try to relate the empirically obtained perceptual saliency values to some factors determining perceptual saliency according to the literature. We consider our data set to be a useful benchmark to test novel hypotheses about perceptual saliency and a reliable normative source to select salient points for future studies with stimuli derived from them (e.g., straight-line and fragmented versions). Therefore, we will present the descriptive, summary statistics and analyses in this article and we will make all of our stimuli and data available on our Web site for future use by the whole research community (see ppw.kuleuven.be/labexppsy/johanw/index.htm).

\section{METHOD}

\section{Subjects}

One hundred sixty-one subjects, all second-year psychology students at the University of Leuven, participated in this study as a mandatory component of their curriculum. It took place in two adjacent rooms with 33 personal computers in total. There were six sessions, with a maximum number of 30 students participating in each session.

\section{Stimuli}

The stimulus set consisted of 260 shapes in outline, derived from the 260 line drawings of everyday objects by Snodgrass and Vanderwart (1980). To convert the line drawings to outline shapes we proceeded as follows: First, silhouettes were made by filling in the interior surfaces in black, and their outlines were then extracted automatically and spline-fitted to obtain smooth curvature values at all points along the contour (for more details, see Wagemans et al., 2007). Because 260 stimuli would be too much for one subject to handle in a single session of a half hour or so, we divided the stimuli into four equivalent subsets (see the Appendix), with a maximally matched distribution of the number of inflections, the number of living/nonliving objects, and the extent to which the outlines could be identified (based on Wagemans et al., 2007; see also De Winter \& Wagemans, 2004).

All black, smoothly curved contour stimuli were presented on a white background at a viewing distance of approximately $0.7 \mathrm{~m}$ in the center of a 17-in. CRT display. The display resolution was set to $1,024 \times 768$ pixels and a refresh rate of $60 \mathrm{~Hz}$. Stimuli were all contained in a box of $640 \times 480$ pixels, or a viewing angle of $16.3^{\circ} \times 12.2^{\circ}$.

\section{Procedure}

Each subject received one page with written instructions and a set of 65 contour stimuli, derived from real objects, as described above. On average, each outline was thus marked by 40 subjects, who were instructed to look carefully at each stimulus and to mark important or salient points. This task was described in the following manner: "Your task is to look carefully at each form and to indicate the points on the outline that are important. Important points are, for instance, points that attract your attention, but also points that can allow the reconstruction of the form." These instructions served no other purpose than to clarify what we mean by salient or important points. The reference to attention and reconstruction makes it easier for subjects to imagine what they could use as a basis for their judgment; it was by no means intended to replace more objective experimental tasks using attention or reconstruction paradigms (see General Discussion).

To ensure that subjects first looked at the outline as a whole, they were allowed to mark points on the outline only after $1 \mathrm{sec}$. A computer program assisted them in selecting the most salient points along the contour; then, using a computer mouse, subjects moved a blue diagonal cross freely on the screen but positioned on the outline shape. The blue cross followed the mouse pointer on the screen so that the Euclidean distance between the mouse pointer and the blue cross was minimal. To mark a point on the outline, subjects moved the blue cross in the desired position and then clicked the left mouse button once. At that position a small (3-pixel radius) blue filled circle became clearly visible and remained there. Subjects could remove a marked point by moving the blue cross to the neighborhood of a marked point (the blue cross then became a red cross indicating the selection of that point) and clicking the left mouse button once. Subjects had to mark at least one point so that they could not inadvertently skip an outline, but they could mark as many as they considered necessary. Instead of enforcing an equal number of points (e.g., 10 points in the study by Norman et al., 2001), we preferred to leave this to our subjects to decide, because our stimulus set varied considerably in shape complexity (e.g., as parameterized by the number of inflections, the number of times curvature changes sign). Each outline remained on the screen for as long as the subjects required - for at least $5 \mathrm{sec}$, again to avoid subjects rushing through the stimuli. The next outline appeared after the Return key was pressed. The position of each marked point was then recorded in data files. The order of stimulus presentation was randomized for each subject separately and the experimenter secured silence throughout the session until the last subject was finished (i.e., after 35-40 $\mathrm{min})$.

\section{RESULTS AND DISCUSSION}

\section{Descriptive Statistics and Data Analysis Procedures}

Data from individual subjects consist of contours (lists of $x$ and $y$ coordinates) with a number of points marked as salient. The number of points marked along the contour differs enormously between stimuli and subjects, with a grand average of 19.8 points $(S D=7.4)$. The average number of points marked by each subject (averaged across stimuli) varies between 3.6 and 47.7 points $(S D=7.4)$, with a median of 19.6 points $\left(Q_{1}=14.4, Q_{3}=24.0\right)$. The average number of marked points per stimulus (averaged across subjects) varies between 3.6 and $66.0(S D=11.2)$, with a median of 15.8 points $\left(Q_{1}=11.0, Q_{3}=26.6\right)$. The average number of marked points per stimulus correlates quite strongly with the number of inflections per stimulus $(r=.893, p<.0001)$.

The total database consists of all the points along all of the contour stimuli marked by all of the subjects, a total of 205,438 points. This is considerably greater than the 1,440 points in the study by Norman et al. (2001), from 12 subjects copying 12 stimuli using 10 points each. One way to test Attneave's (1954) hypothesis is to investigate how far each marked point is from the different types of curvature singularities: positive maxima $(M+)$, negative minima $(m-)$, and inflections $(I)$. To calculate this, we took the so-called outline-distance, the Euclidean distance in pixels from point to point, accumulated for all points along the outline between the marked point and the target singularity. When relating each marked point to the nearest exemplar of each of the three curvature singularities (i.e., using each marked point three times), the average distances are 15.65 pixels $(S D=20.29)$ for $M+, 156.70$ pixels $(S D=1,129.26)$ for $m-$, and 152.53 pixels $(S D=$ 
$1,129.50)$ for $I$. When relating each marked point to the nearest singularity only, regardless of which of the three (i.e., using each marked point only once), the majority of marked points $(110,355$ or $53.72 \%)$ was closest to an $M+$ (average distance: 6.93 pixels, $S D=11.10$ ), then $m-$ $(68,053$ or $33.12 \%$, average distance: 4.05 pixels, $S D=$ $5.80)$, and finally $I(27,030$ or $13.16 \%$, average distance: 5.83 pixels, $S D=8.39$ ). However, both of these measures are strongly influenced by the frequency of occurrencewhich also differs strongly - of the three curvature singularities in the stimuli themselves: $34.4 \% \mathrm{M}+, 25.2 \% \mathrm{~m}-$, and $40.5 \% I$. The same order of frequency $(I>M+>$ $m-)$ is also reflected by the average number of singularities of each type per stimulus: For $M+, 22.6(S D=13.3$, median $=18.0)$; for $m-, 16.6(S D=12.9$, median $=$ $13.0)$; and for $I, 26.6(S D=19.7$, median $=22.0)$. When comparing the attraction of the three types of curvature singularities in the analyses reported below, we can compensate for this predominance of $I$ and $M+$ over $m-$ by dividing by these relative frequencies.

Instead of considering individually marked points, we can use the frequency of marking in each point along the contour as the major dependent variable. These raw frequency values vary between zero (when none of the participants marked a given point) and about 40 , the number of participants for that stimulus (when all the participants marked a given point). The distribution of these frequency values is very strongly left-skewed: The majority of points - over 50,000 - are marked only once, about
20,000 points are marked twice, about 10,000 points are marked three times, and so on, with a very long right tail (a few points with a relatively high frequency). A second way to test Attneave's (1954) hypothesis, then, is to compare the frequencies of marking between the three subsets of marked points: one where an $M+$ is the nearest neighbor, one where an $m$ - is the nearest neighbor, and one where an $I$ is the nearest neighbor (see Figure 2A). As soon as we look at points marked at least twice, it is very clear that those closer to extrema (i.e., $M+$ and $m-$ ) are much more frequent than those closer to inflections $(I)$. When we correct for the larger frequency of occurrence of $I$ relative to $M+$ and $m-$ in the stimuli themselves (as indicated above), the difference becomes even stronger. The same is true of points marked only once (see Figure 2B).

It is possible to plot these frequency values along with the curvature graph of each contour (see Figure 3Ablack and gray lines, respectively) and we can then take the local maxima of these saliency graphs; we call these the saliency maxima. A third way to test Attneave's (1954) hypothesis now becomes to investigate how far these saliency maxima are located away from the three different types of curvature singularities (taking the outlinedistance between each saliency maximum and the target singularity).

However, two undesired effects emerged from the above procedure. First, the distributions of saliency maxima are always quite erratic. Two saliency maxima are often located close to each other. It seems reasonable to assume
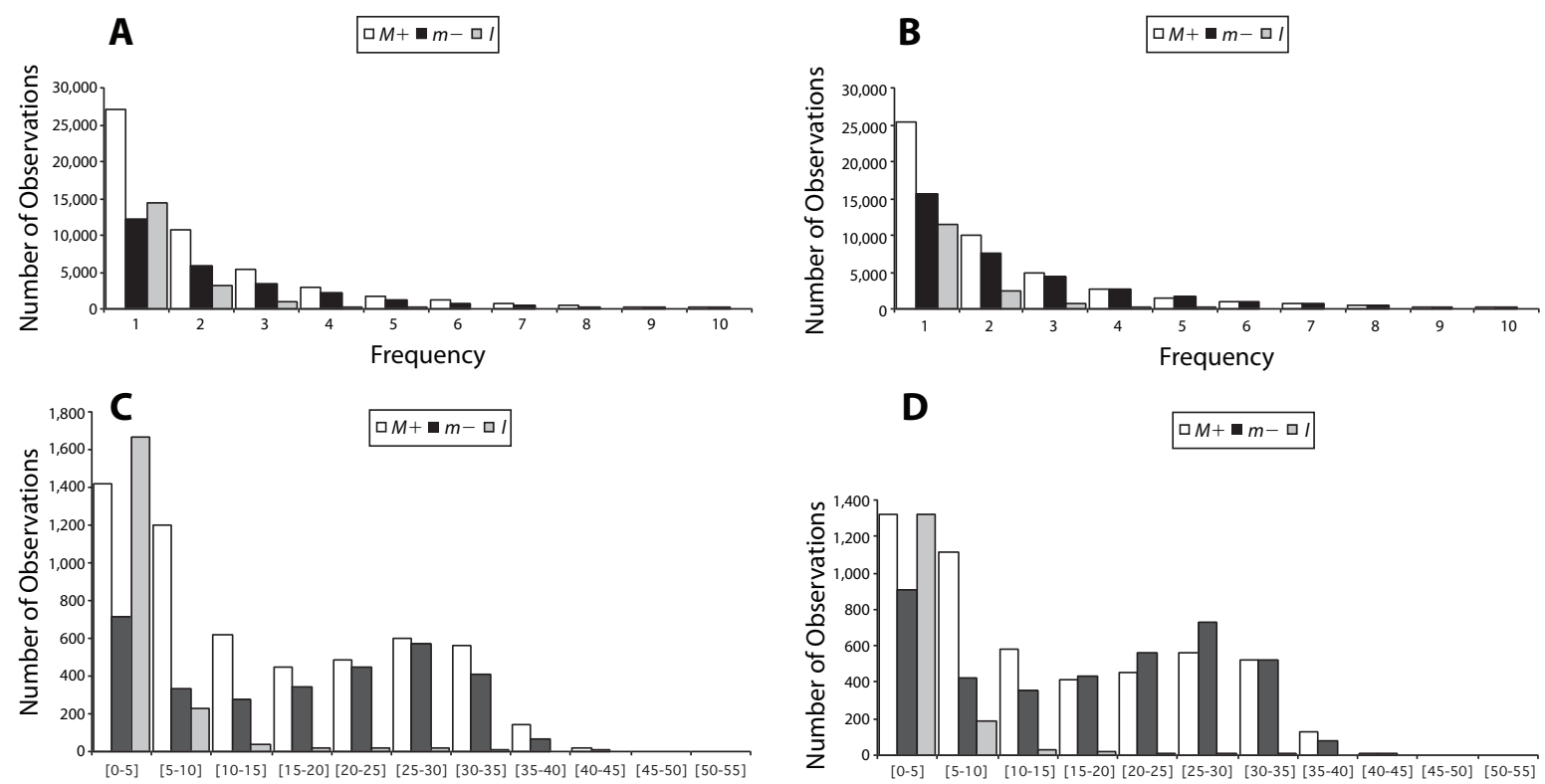

D

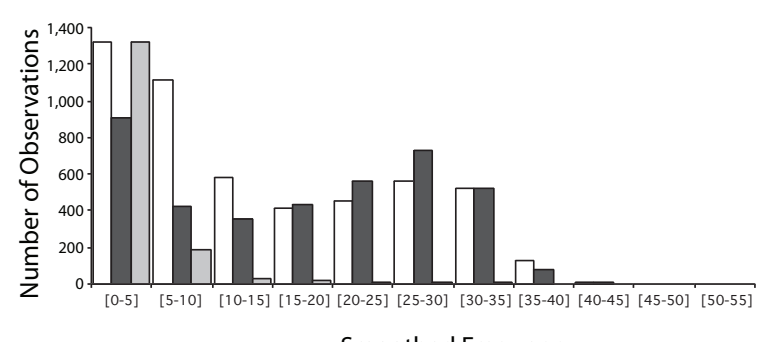

Smoothed Frequency

Smoothed Frequency

Figure 2. Histograms of raw and smoothed frequency values of marked points being closest to maxima $(M+)$, minima $(m-)$, or inflections $(I)$. In (A) the uncorrected raw frequency values are plotted, whereas in (B) these values are corrected for the relative frequency of occurrence of each type of curvature singularity (see text for further details). Raw frequencies of two or higher occur mainly for points associated with extrema, not with inflections. In (C) the uncorrected smoothed values are plotted, whereas in (D) these values are corrected for the relative frequency of occurrence of each type of curvature singularity (see text for further details). The highest frequencies relate to extrema, not inflections. 
A
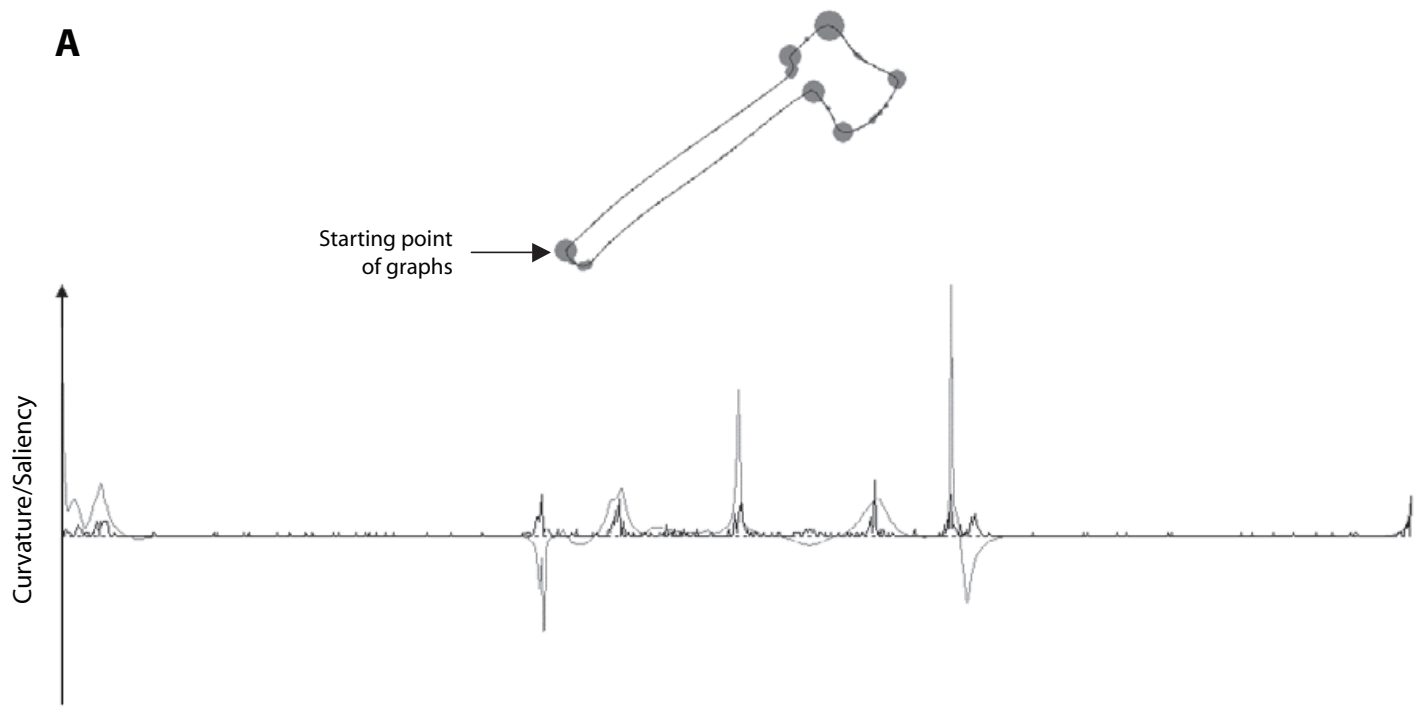

B
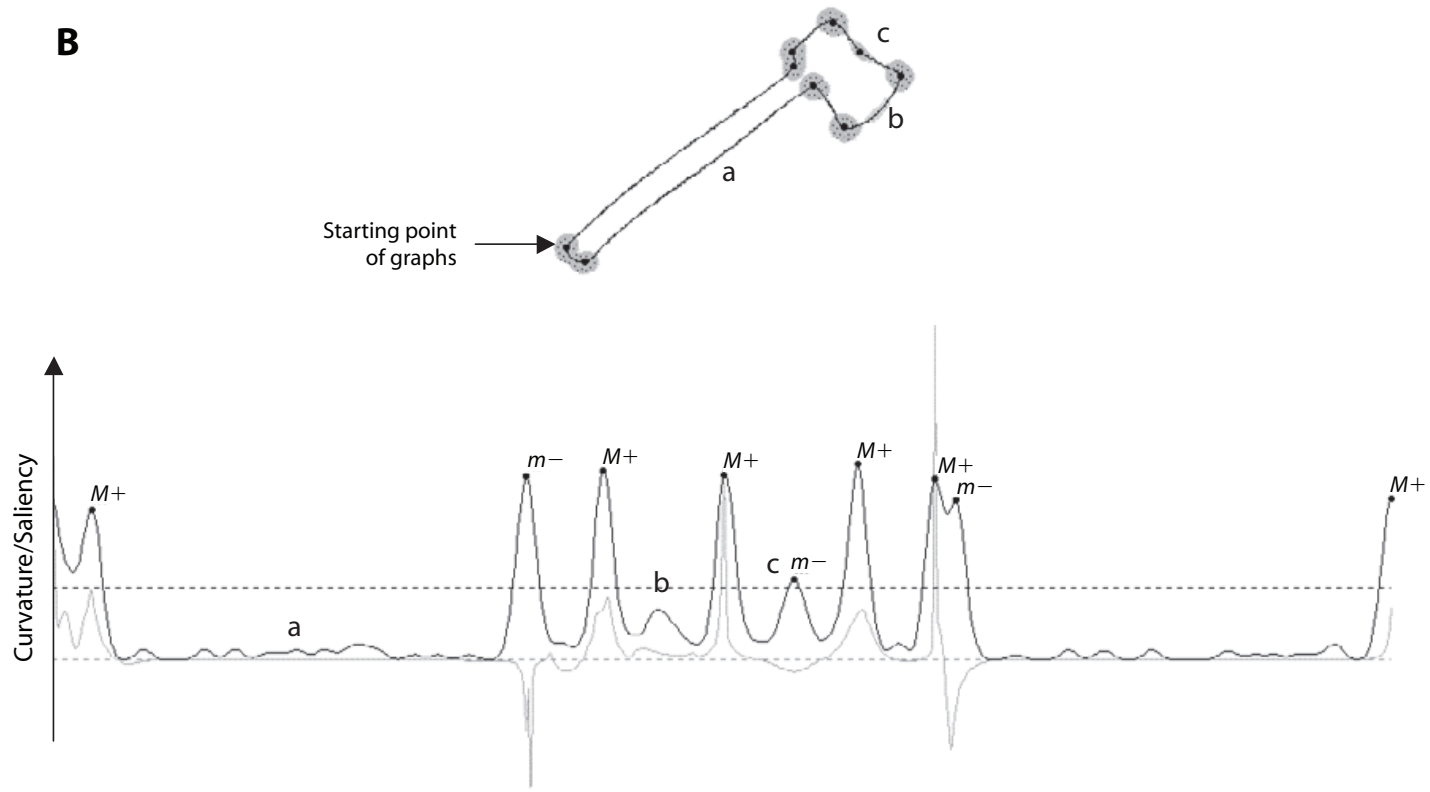

Figure 3. (A) Stimulus example (No. 12, axe) with the raw frequencies of marked points superimposed on the contour (the size of the gray circle is proportional to the frequency). Below the stimulus example, the curvature graph is plotted in gray, and the raw frequency is plotted in black. The contour is traced counterclockwise, starting at the left corner point of the bottom part of the handle (indicated by the arrow). The curvature maxima and minima are clearly visible and appear to be attractive locations to be marked as salient points. (B) Same stimulus example and curvature graph but now with smoothed saliency being superimposed (see text for details). The selection of the specific smoothing parameters determines whether some points will be selected as salient points or not (see text for details). Many instances of selected saliency maxima (indicated by solid black dots) are situated at positive maxima $(M+)$ or negative minima $(m-)$.

that such near neighbors do not reflect two independent salient points, but have become two points due to slight deviations in the marking location. To avoid this undesired result, we applied smoothing to the saliency frequencies. The smoothing technique calculated for each point $i$ the weighted average of the frequency of point $i$ and of points in the neighborhood of $i$. The weighting was done by a
Gaussian function; the farther the points are away from point $i$, the less influence they have on the saliency value of point $i$. We used a Gaussian function, because we considered it more and more unlikely that a subject meant to mark point $i$ the farther it was away from point $i$, and we assumed this deviation or noise to be distributed according to a Gaussian function. An important parameter is the 
width or standard deviation of the Gaussian function. This smoothing parameter is expressed in image pixels. So, after this smoothing, we had a smoothed saliency value for every point on the contour: The higher this value, the more subjects chose that point (or points nearby) as salient (see Figure 3B). A second problem is that some (very) low value saliency maxima occur (e.g., point $a$ in Figure 3B); it seems inappropriate to treat these points as salient points too, considering their low value. Therefore, we applied a saliency threshold, a simple cutoff: All points below a particular saliency value are no longer considered. It will be clear that the selection of an appropriate threshold value is not a trivial matter. In the example of Figure 3B, a lower threshold value would have included point $b$, whereas a slightly higher threshold value would have missed point $c$.

To summarize: (1) we smoothed the saliency frequencies; (2) we obtained local maxima points of these smoothed saliency values; (3) we selected the saliency maxima above the saliency threshold (or equal to it), and considered these points as the selected salient points; and (4) we examined what the nearest neighboring curvature singularities were for each of the selected salient points. The problem was then to choose appropriate values for these two parameters, the smoothing parameter and the saliency threshold. We therefore decided to test a wide range of parameter values and to examine their effect. This constitutes the basis of the results reported in the second paragraph below. To avoid overloading the article with data, we will choose a range of parameter values that represent the general trends in our data, and we will limit ourselves to parameter values with fewer artifacts (e.g., floor-ceiling effects). A substantial part of the article will address the variability due to these parameter changes.

To conclude this more descriptive paragraph, we include histograms for the three subsets of marked points, as in Figures 2A and 2B, but now for the smoothed frequency (taking a smoothing parameter of 5 pixels and no threshold): Figure $2 \mathrm{C}$ contains the histograms for the smoothed frequencies for marked points closest to $M+, m-$, and $I$ as such, and Figure 2D corrects the number of observations by compensating for the larger frequency of occurrence of $I, M+$, and $m-$ in the stimuli themselves (see above). These graphs clearly show that high values of smoothed frequency (or saliency) occur almost exclusively at points that are closest to extrema, not at inflections. For moderate values of smoothed saliency (e.g., 5-15), points closest to $M+$ are somewhat more frequent than points closest to $m-$, whereas the reverse is true for relative high values of smoothed saliency (e.g., 20-30).

\section{Selected Salient Points and Distance to Curvature Singularities}

In line with the third approach to test Attneave's (1954) hypothesis (introduced above), we will now examine more closely the set of salient points as marked by our observers (i.e., selected saliency maxima), and we will specifically investigate how they relate to the curvature singularities as computed mathematically on our contour stimuli. Several procedures will be used that will all lead to more or less the same conclusion - that, in general, highly salient points are very often close to the curvature extrema, as predicted by Attneave.

In a first analysis, we take all of the selected salient points, using all different combinations of seven smoothing values (from 2 to 14 in steps of 2) and seven saliency thresholds (from 11 to 29 in steps of 3 ), and evaluate whether they are closest to an $M+$, an $m-$, or an $I$. To correct for the uneven occurrence of the three curvature singularities (see above), we express the frequency of a selected salient point being the closest to a curvature singularity of a particular type, relative to the frequency of occurrence of that singularity type. Figure 4A plots such frequencies for the whole range of parameter values, separately for $M+$ (squares), $m-$ (triangles), and $I$ (circles). It is clear that extrema are selected most frequently, in accordance with Attneave's (1954) hypothesis, with negative minima $(\mathrm{m}-)$ being selected somewhat less frequently than positive maxima $(M+)$, especially with a relatively large smoothing parameter. This difference tends to become smaller with a higher saliency threshold (i.e., including only the highly salient points). At the same time, inflections $(I)$ tend to become more frequent nearest neighbors of selected salient points with larger smoothing values. These observations are supported by an ANOVA: The relevant main effects and interaction effects are all strongly significant $(p<.0001)$, with singularity type clearly being the strongest factor $[F(2,72)=1,202.82]$, explaining more than $88 \%$ of the systematic variance.

In a second analysis, we took all of the selected salient points, for all the same combinations of seven smoothing values and seven saliency thresholds, and plotted the distance to its closest curvature singularity, an $M+$, an $m-$, or an $I$ (see Figure 4B). This outline-distance is generally largest for $M+$ points, smallest for $I$, and intermediate, but relatively smaller, for $m-$. For larger smoothing values, the distance tends to become larger. This quasilinear increase is strongest for $M+$ points when the saliency threshold is lower (thereby also considering the lower value saliency points), whereas it is fairly constant for $m-$ and $I$. Again, these trends are confirmed by an ANOVA: The relevant main effects and interaction effects are all strongly significant $(p<.0001)$, with singularity type clearly being the strongest factor $[F(2,72)=756.02]$, explaining more than $58 \%$ of the systematic variance.

While exploring several outline shapes visually, we noticed that for larger contour segments which are almost uniformly (mostly positive) curved, the marked points tend to spread out more. There is much less agreement as to which point is most salient on such segments (see Figure 5). A good example of this is the positively curved side of the banana (A). In shapes with these large convex sections, factors others than those based on local curvature changes come into play as well. For example, the selection of four points in the case of the ball (B) is clearly determined by the horizontal and vertical axes, whereas the four selected points in the American football (C) indicate the intersections of the long and short axes of the 2-D ellipse (from the projected 3-D ellipsoid) with the contour. Subjects have the tendency to spread out their 
A

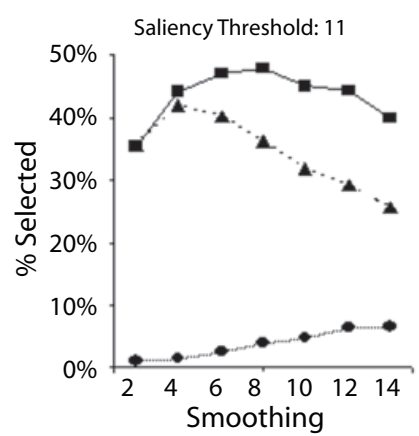

B

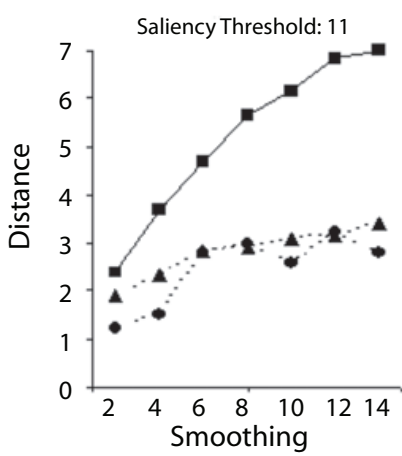

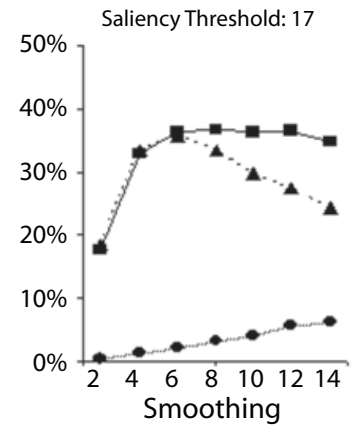
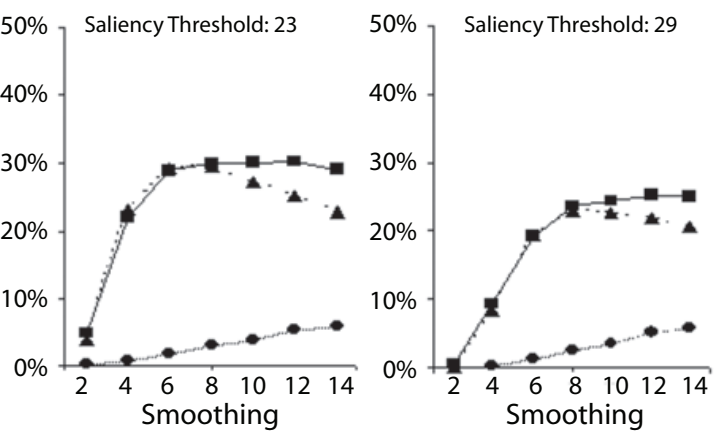

$\rightarrow-M+\cdots \cdot m-\cdots \cdot-1$
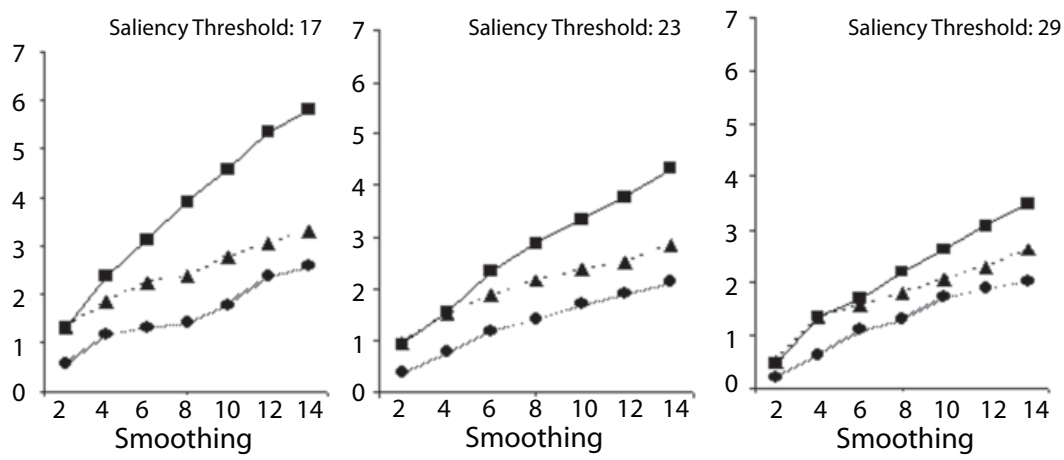

Figure 4. (A) Frequency of selected salient points being closest to maxima $(M+)$, minima $(m-)$, or inflections $(I)$, expressed relative to the frequency of occurrence of each type of curvature singularity. The different panels report these results for different saliency thresholds (from 11 to 29 in steps of 6), whereas the values on the $x$ axes indicate the different smoothing values (from 2 to 14 in steps of 2). These smoothing parameters have some effect on the frequency values but clearly $M+$ and $m$ - are always much more strongly represented than $I$. (B) Distance between all the selected saliency maxima and their nearest singularity, shown separately for each type of curvature singularity $(M+, m-$, or $I)$. The saliency thresholds (in the different panels) and smoothing values (on the $x$ axes) are the same as in panel $A$. These smoothing parameters have some effect on the frequency values but for relatively small smoothing values ( 2 to 6 ) and for relatively large saliency thresholds (retaining only the most salient points), the distances to singularities are small (e.g., up to 4 pixels).

markings somewhat evenly along these contour segments, a tendency that may have played a role in Kennedy and Domander's (1985) finding that fragments at midpoints between curvature singularities are helpful for identification (see also Kennedy, Juricevic, \& Bai, 2003).

Segments of quasiuniform curvature are also more susceptible to the chosen parameter values. In the example of the alligator (see Figure 5D), we gradually decreased the value of the saliency threshold from 10 to 8 and 6 (holding the smoothing value constant at 6). Observe that with each chosen saliency threshold, additional saliency points appear (from 28 at the highest threshold to 31 at the intermediate threshold and 34 at the lowest threshold). The newly appearing points are usually located at homogeneously curved sections along the contour (e.g., the back and the right side of the tail of the alligator). This illustrates again how difficult it is to select an appropriate saliency threshold if one wants to select representative salient points (e.g., to be used in future experiments with straight-line versions or fragmented versions). It can be concluded from these results that it is best to select a smaller smoothing value (but as small as, say, 5) to avoid artifacts. In that case, it is clear that extrema are the most salient points on an outline shape and that $M+$ and $m-$ points are chosen in approximately equal amounts. Inflections are rarely chosen as salient points.

This can be illustrated quite convincingly using representative examples of our stimulus set, superimposing the most frequent markings on the contours along with the curvature singularities (using a data format similar to Figure 5 in Norman et al., 2001). In Figure 6, 12 examples are shown of these marked and annotated object contours (all 260 are available on our Web site): These examples are representative in the sense that they show animals as well as artifacts, relatively simple shapes as well as more complicated ones, and they contain many typical instances of marked singularities, as well as some nonmarked singularities and marked nonsingularities. The procedure to obtain these example figures is the following: First, the smoothing value is set to 5 pixels and the saliency threshold is set to 13.3 pixels (i.e., the average saliency across the whole stimulus set). Second, the extrema are shown by green squares for $M+$ and red triangles for $m-$, the size of the symbol being related to the absolute 
A

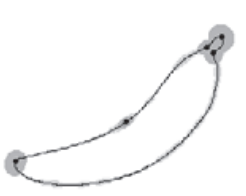

D

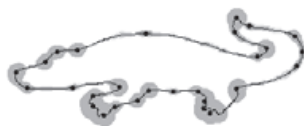

B

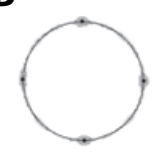

C

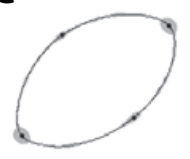

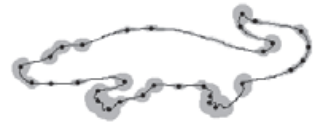

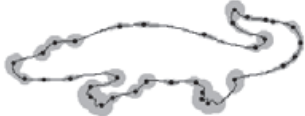

Figure 5. Stimulus examples with smoothed saliency values superimposed on the contours (in gray) and selected saliency maxima indicated by solid black dots. (A) Banana (No. 16), (B) ball (No. 14), and (C) American football (No. 95). These examples show that contour segments with smooth, quasihomogeneous positive (convex) curvature often yield saliency values that are spread out, which make it more difficult to reach the threshold. (D) Alligator (No. 3). The number of selected saliency maxima (marked by solid black dots) increases from 28 to 31 and 34 when the saliency threshold decreases from 10 to 8 and 6.

curvature at that point (curvature is multiplied by 20 pixels, but the maximum radius is set to 7 pixels). In between the selected extrema, the inflections are indicated as blue circles with fixed radii of 3 pixels. Third, the radii of the open circles, indicating the selected marked points, are related to the saliency (radius = saliency/4). In many examples, the most salient points are located at the strongest curvature extrema (positive maxima as well as negative minima). This visualization of the results is probably the most convincing evidence in favor of Attneave's (1954) hypothesis.

\section{Factors Determining the Saliency of Marked Points}

In this part of the article, we will examine possible factors that might determine the saliency of the points along the contour marked by our subjects. Attneave's (1954) hypothesis concerned only a simple binary distinction between curvature extrema and other points (the former being salient, the latter not) and Norman et al.'s (2001) article also did not report the role of other factors underlying the empirically obtained saliency values. As our preceding descriptive results have indicated, curvature extrema are often marked as salient points; but certain other points are salient too, and clearly not all curvature extrema are equally salient. We want to understand some of the variability in our saliency measure in terms of contributing stimulus factors. The present analysis is basically correlational in nature and the results will have to be followed up by future psychophysical experiments that are specifically designed to test the role of the putative factors parametrically (using appropriate controls for possibly confounding factors). Despite the post hoc nature of these analyses, however, we believe it is useful to report some of these results because they might add insight into what determines the saliency of a point along the contour of an object. Several of these post hoc analyses address issues raised in the literature, whereas others are based on our own intuitions.
Feldman and Singh (2005) proved mathematically that points of negative curvature are more informative than are points of positive curvature, because they are more surprising and less redundant. Whether this higher information value, which was based only on local factors (except for the assumption of closure), is also reflected by a higher perceptual saliency, as marked by our participants, can be verified in our large data set of saliency values. One way to address this question is to look at the relation between absolute curvature and smoothed saliency (using a fixed smoothing parameter of 5 pixels and a threshold of 0 ) and do this separately for contour points with positive and negative curvature. The linear regression through these data points reveals a much steeper slope for the positively curved points (20.20) than for the negatively curved points (4.73), with a comparable intercept (11.32 and 13.81, respectively). This seems to provide evidence against the link between information value and saliency: For an equal value of absolute curvature, the saliency is higher for positively curved points than for negatively curved points. However, the fit of these linear regressions is relatively poor $\left(R^{2}=.163\right.$ and .043 , respectively), and the results may be strongly influenced by some outliers. Restricting the range to absolute curvatures smaller than, or equal to, 1, yields a similar pattern, although somewhat less pronounced: The slope for the positively curved points (67.30) is somewhat steeper than for the negatively curved points (54.48), with a comparable intercept (8.92 and 10.29 , respectively), and reasonable fits this time $\left(R^{2}=\right.$ .407 and .339 , respectively). The conclusion must be that points with negative curvature are not necessarily more salient than points with positive curvature, despite their higher information value. This conclusion is perhaps not very surprising, if one considers that Feldman and Singh's analysis of information value was purely mathematical and local, whereas our saliency values are subjective and based on a much more global perceptual analysis of shape. In other words, we can conclude that pure mathematical 


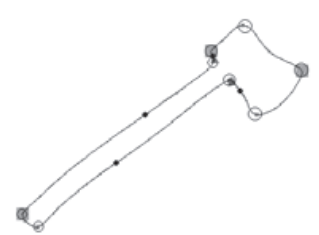

$012-$ Axe

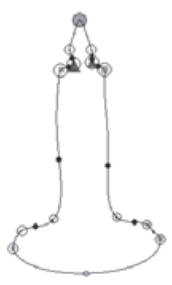

044 - Candle

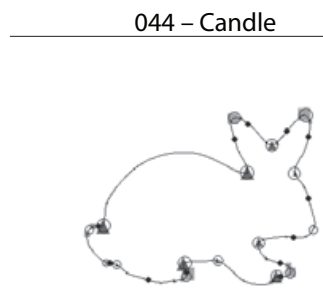

182 - Rabbit

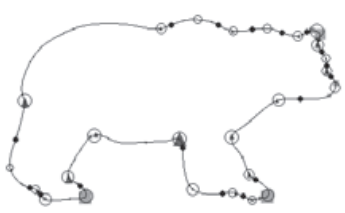

021 - Bear

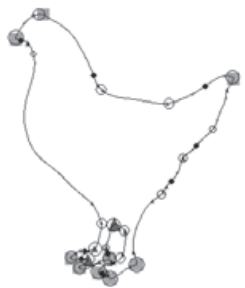

055 - Chicken

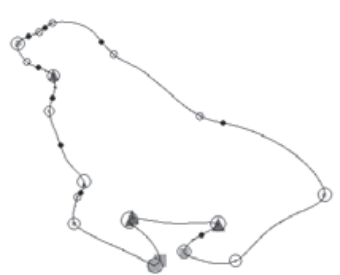

201 - Seal
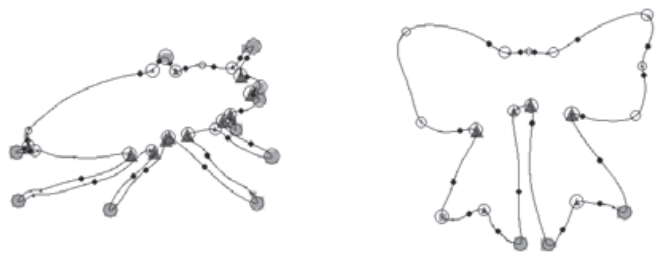

024 - Beetle

033 - Bow
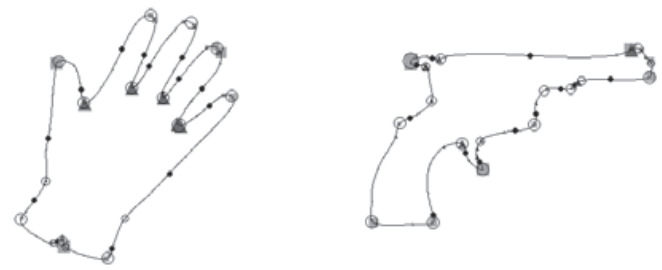

112 - Revolver

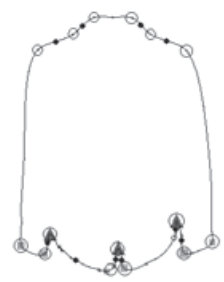

$203-$ Shirt

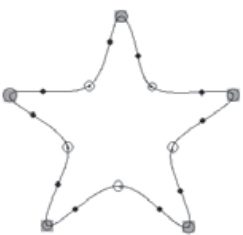

$217-$ Star

Figure 6. Stimulus examples (indicated by their names and numbers within the set by Snodgrass and Vanderwart, 1980) with curvature singularities and selected saliency maxima superimposed on the contour. Squares indicate $M+$, triangles indicate $m-$, and dots indicate $I$. Open circles indicate selected saliency maxima. The size of the symbols reflects their absolute curvature (for extrema) or their saliency (for saliency maxima). This figure can be compared more or less to Figure 5 in Norman et al. (2001), also illustrated as Figure 1C before. The complete set of stimuli and results is made available on our Web site (see ppw.kuleuven.be/labexppsy/johanw/ index.htm).

information (in terms of curvature sign and value) was not the only factor influencing selection by the participants.

For all of the remaining factors, we will report the correlation between the parameterization of the factor (as computed on our stimuli) and the empirically determined saliency values after smoothing. We will plot these correlations for all different combinations of seven smoothing values (from 2 to 14 in steps of 2) and seven saliency thresholds (from 8 to 26 in steps of 3 ), as well as the smoothed data without a threshold (threshold $=0$ ). These plots are included in Figure 7 (using different panels for the different factors being examined). We will make use of some additional figures to illustrate the specific procedures used to compute the relevant stimulus factors (see Figure 8).

A simple factor to examine is the absolute curvature of a point. As can be seen in Figure 7A, this factor produces generally low correlations (between .20 and .35 when no threshold is applied, lower than .20 in most other cases) and they decrease with larger smoothing values and higher saliency thresholds. One reason that absolute curvature may not be such a strong factor is that it can vary a lot from one point to another, especially in contours derived from natural objects (due to discretization). A strong cur- vature peak with limited spatial extent will not be very salient visually. Therefore, rather than using absolute curvature as such, it is useful to normalize the absolute curvature by some measure of the extent of the shape in the neighborhood of the point. In their work on the saliency of a part boundary (usually an $m-$ ), Hoffman and Singh (1997) proposed to divide the curvature of a point by the length of the chord connecting the two neighboring inflections (see Figure 8A). As can be seen in Figure 7B, this normalization does not improve the correlations. They are still generally low (only slightly above .20 when no threshold is applied, lower than .20 in all other cases), they decrease with higher saliency thresholds, but they now reach a maximum for smoothing values around 4 or 6 . As an alternative basis for normalization, we can take the chord length between the two midpoints in between the selected salient point and each of its two neighboring salient points (see Figure 8B). As can be seen in Figure 7C, this alternative normalization yields larger correlations for the smoothed saliency values without a threshold (above .40 when the smoothing parameter is not larger than 8), but as soon as a threshold is applied, the correlations are around .30 at best (for smoothing parameters, around 4 or 6 ). 

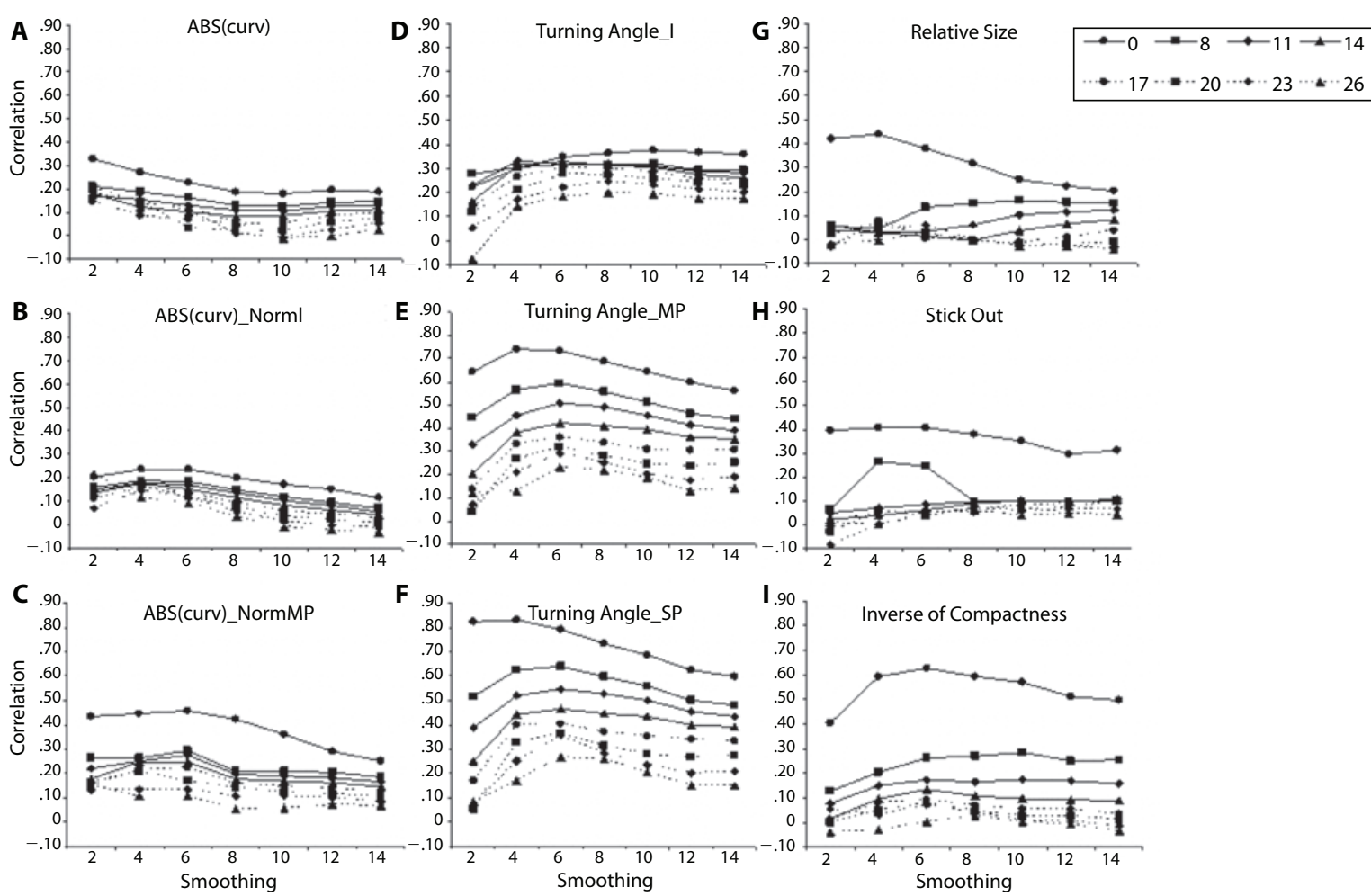

Figure 7. Correlations between stimulus factors and saliency for different smoothing values (along the $x$ axes) and for different saliency thresholds (shown as different lines). Panels (A) to (C) show the results for different measures of absolute curvature at a local point, (D) to (F) for different calculations of the turning angle between the contour segments neighboring the selected target point, and (G) to (I) for more global part measures (see text for further details).

The generally weak correlations between curvature and saliency are consistent with neurophysiological research (Pasupathy \& Connor, 1999) showing that cells in monkey area V4 are more tuned to specific angles between two adjacent lines of a corner and less to the degree of curvature at the apex of the angle - although both data sets are quite different in many respects (e.g., humans vs. monkeys, subjective judgments vs. cell responses). In relation to this, Hoffman and Singh (1997) proposed the turning angle as another factor determining the saliency of a part boundary, which we might extend to the saliency of a marked point. The turning angle is simply the amount of turning from one normal to another normal (using the angle smaller than $180^{\circ}$ ). Rather than taking the normal, we calculated an estimate by fitting a regression line through the target point and its 10 surrounding contour points ( 5 at each side), and we then used its slope to compute the turning angle. This procedure has the advantage of being less sensitive to small local irregularities. Again, we can use different basis points of line segments to compute the turning angle between. Similar to Hoffman and Singh's measure, we can use the two neighboring inflections of each salient point (see Figure 8C). Similar to our preceding normalization procedure, we can also use the midpoints between two consecutive salient points (see Figure $8 \mathrm{D}$ ), or we can extend the local neighborhood even further and connect the neighboring salient points with line segments (see Figure 8E). The disadvantage of taking our saliency-based reference points is that they depend on the smoothing parameters (e.g., with a different saliency threshold, the neighboring salient points will change).

The respective correlations for these three measures based on turning angle are shown in Figures 7D, 7E, and $7 \mathrm{~F}$. The turning angle based on neighboring inflections (see Figure 7D) yields correlations that are generally low (between .15 and .30) and do not vary much with the smoothing parameters. Our variants of the turning angle, using the neighboring salient points (see Figure 7F) or the midpoints between them (see Figure 7E), produce the highest correlations of all of our measures, especially when no threshold is applied (between .60 and .85). In line with the neurophysiological results reported earlier (Pasupathy \& Connor, 1999), we have strong evidence that the turning angle between the two flanking line segments of a corner is an important factor for the perceptual saliency of the corner point-more important than the local curvature in the corner point itself. Note that subsequent neurophysiological work has indicated that higher level shape encoding in the monkey cortex makes use of lower level encoding of curved fragments (Pasupathy \& Connor, 2001, 2002), and may correspond strongly with human shape encoding (Op de Beeck, Wagemans, \& Vo- 


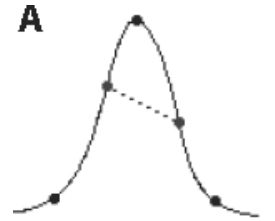

D

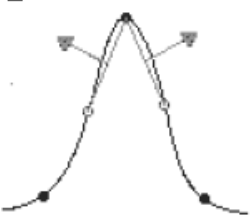

B

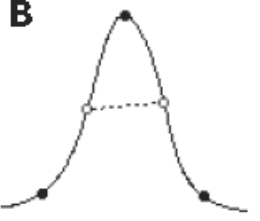

$\mathbf{E}$

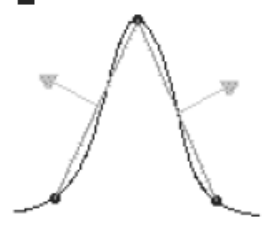

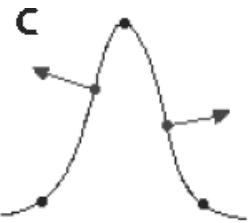

$\mathbf{F}$

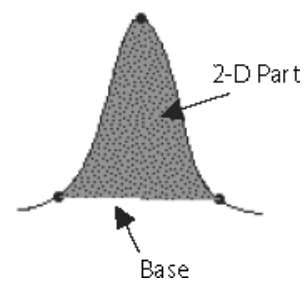

Figure 8. Illustrations of the principles to compute the stimulus factors. (A) Chord length between two inflections (in between two extrema). (B) Chord length between two midpoints (in between two selected salient points). (C) Turning angle measured between the normals at the two inflections neighboring the selected salient point. (D) Turning angle measured between the two straight line segments connecting the selected salient point and the midpoints to the two neighboring salient points. (E) Turning angle measured between the two straight line segments connecting the selected salient point and the two neighboring salient points themselves. (F) Illustration of the 2-D part defined around a selected salient point. Possibly relevant stimulus factors for this 2-D part are relative size, stick out, compactness, and contour fragment length (see text for further details).

gels, 2001, 2003). Our data set shows clearly that a point is considered more salient when its two neighboring line segments form a sharp angle. As expected, the correlations between saliency and our variants of the turning angle depend rather strongly on the smoothing parameters. The curves for the different smoothing parameters now tend to follow an inverse U-shaped function, with peaks (highest correlations) around a smoothing value of 6 pixels. As before, the correlations decrease with higher saliency thresholds but now, in contrast to the results for the other factors, the correlations are still considerable (between .40 and .65) even for thresholds of 8, 11, and 14.

Extending the local neighborhood around a salient point even further (from chord length to normalize curvature in a salient point, to turning angle between two neighboring segments), we then examined the role of the 2-D part of the shape surrounding the salient point. (Note that we use the term "part" in a theoretically neutral way, not in the sense of making any claims about whether the 2-D piece of shape is a perceptually meaningful part or a structural component of the whole shape. The segmentation of shapes into natural parts is not at stake here. This issue has been studied in detail elsewhere; see De Winter \& Wagemans, 2006; Hoffman \& Richards, 1984.) We selected both salient points neighboring the salient point under consideration (as before) and we then connected them with a line segment, constituting a base line. The relevant part around a salient point is now defined as the part of the shape inside the contour bordered by the two neighboring salient points and the base line connecting them (see Figure 8F). Hoffman and Singh (1997) proposed two factors determining the saliency of a part (they used "part" in the stricter sense of natural parts or components). One is the size of the part relative to the whole object (i.e., the area of the gray part in Figure 8F, divided by the area of the whole outline to which it belongs). The second is what they called stick out: the degree to which a part protrudes from the object (i.e., the length of the contour segment of the gray part in Figure 8F, divided by the length of the base). The larger a part, and the more it sticks out, the more salient it is. When we are dealing with salient points of positive curvature, the relevant parts belong to the inside of the object (hence, figural parts). When we are dealing with salient points of negative curvature, in contrast, the relevant parts belong to the outside of the object (hence, background parts or figural holes). To be able to distinguish between the relevance of factors influencing the saliency of parts in both of these contexts, we will report the analyses separately for positive and negative points.

A third factor that we also considered relevant is the so-called "compactness" of the part. Zusne (1970) has defined compactness of overall shapes as the area of the shape divided by the squared contour length. Intuitively, shapes are most compact if their area is as large as possible for the shortest possible contour length. A round shape is very compact, whereas a shape with a lot of parts sticking out has a low compactness. We hypothesize that the saliency of a part may be related to the inverse of compactness (i.e., fewer compact parts are more salient in the 
sense that they stick out more). We therefore define the inverse of compactness of our parts as the squared length of the contour of the part (i.e., contour segment from each neighboring salient point to the salient point under consideration, plus the length of the base), divided by the area of the gray part in Figure 8F.

The respective correlations for these three measures based on the part surrounding the salient point (i.e., relative size, stick out, and inverse of compactness) are shown in Figures 7G, 7H, and 7I. The two measures affecting part saliency, according to Hoffman and Singh (1997) relative size and stick out-yield rather weak correlations with point saliency in our data set, except for unthresholded saliency values with a small smoothing value. For relative size (see Figure 7G), the correlations for the unthresholded saliency values drop from around .40 and .45 (for a smoothing parameter of 2 and 4 pixels, respectively) to about .20 (for a smoothing parameter of 12 or 14 pixels). For stick out (see Figure $7 \mathrm{H}$ ), the correlations for the unthresholded saliency values are more stable for an increasing smoothing parameter (from .40 to about .30). All the other correlations with stick out are low, except for two correlations around .25 or .30 for a smoothing parameter of 4 or 6 pixels and a threshold of 8 pixels. When we tested the correlations separately for points with positive and negative curvature (for those parameter values for which the correlations are maximal in the overall set, i.e., a smoothing parameter of 4 pixels and no threshold), they turned out to be quite stable: for relative size ( $r=.44$ and .45 for parts surrounding a point with positive and negative curvature, respectively; both $p \mathrm{~s}<.05$ ), and for stick out $(r=.43$ and .36$)$, respectively; both $p$ s $<.05)$. The saliency of a point is therefore affected more or less equally by the saliency of the surrounding part, whether the part is positive (protrusions) or negative (indentations). As expected, the inverse of the compactness of the surrounding part also turns out to affect the saliency of a point (see Figure 7I): For the unthresholded data, the correlations vary between .40 and .60 (with a peak at a smoothing parameter of 6 pixels). Points are more salient when the parts in which they are embedded are less compact.

Because it is quite likely that some of the tested factors correlate with one another, it is also useful to perform an overall multivariate linear regression with all $10 \mathrm{fac}-$ tors that have been tested (i.e., sign of curvature and the nine factors plotted in Figure 7). To keep the reported data within a manageable amount, we performed this analysis for only one combination of parameter values: a smoothing value of 4 pixels and a threshold of 0 . These values were chosen because they generally produced the largest correlations (see Figure 7). The results of this regression analysis are given in Table 1 . The $R^{2}$ for the overall model, including all 12 factors, was .74. The majority of the explained variance can be attributed to our third measure of line turning (taking neighboring salient points to define the line segments; see Figure $8 \mathrm{E}$ for the definition and Figure $7 \mathrm{~F}$ for the results): $.79 \times .79=.62(=83.78 \%$ of the systematic variance). Also important are stick out and compactness, each contributing $-.22 \times-.22=.048$ $(=6.47 \%)$ and $-.17 \times-.17=.029(=3.91 \%)$, respectively. Adding our normalized absolute curvature measure (using midpoints between salient points rather than inflections) adds about $1 \%$ explained variance; so all in all, although we are analyzing factors determining the perceptual saliency of points along the contour, the more global factors seem to be more important than the local ones.

\section{GENERAL DISCUSSION}

Which points along the contour of a 2-D shape are most salient? This simple question was the starting point of the present study. More than half a century ago, Attneave (1954) proposed that local maxima of positive curvature and local minima of negative curvature are the most salient points along the contour. Subsequent mathematical work has substantiated Attneave's intuition by explaining its geometric basis (e.g., Koenderink, 1984) and by proving that curvature extrema are indeed more informative in the sense of information theory (Feldman \& Singh, 2005; Resnikoff, 1989). The empirical support, however, was less strong so far. Norman et al. (2001) were the first to show empirically obtained saliency data along with mathematically computed curvature data but their study used only 12 observers and 12 quasirandom shapes (shadows cast by sweet potatoes). Using identification of contour-deleted line drawings of everyday objects, Kennedy and Domander (1985) had shown that points midway between extrema were more informative than the extrema themselves.

Table 1

Results From the Stepwise Multiple Regression Analysis of Smoothed Saliency Against 10 Stimulus Factors

\begin{tabular}{lrrrrrr}
\multicolumn{7}{c}{ Saliency Against 10 Stimulus Factors } \\
\hline & $\beta$ & $S E$ & $\beta$ & $S E$ & $t(12,360)$ & $p$ level \\
\hline Intercept & & & 8.19 & 0.17 & 49.35 & .0000 \\
Turning angle_SP & 0.79 & 0.01 & 0.21 & 0.00 & 70.19 & .0000 \\
Stick out & -0.22 & 0.01 & -2.64 & 0.08 & -33.88 & .0000 \\
Compactness & -0.17 & 0.01 & -0.03 & 0.00 & -26.62 & .0000 \\
ABS(curv)_NormMP & 0.12 & 0.01 & 0.40 & 0.02 & 16.17 & .0000 \\
Turning angle_MP & 0.09 & 0.01 & 0.02 & 0.00 & 10.18 & .0000 \\
Turning angle_I & -0.07 & 0.00 & -0.01 & 0.00 & -14.63 & .0000 \\
Relative size & 0.06 & 0.01 & 0.95 & 0.10 & 9.80 & .0000 \\
ABS(curv) & -0.05 & 0.01 & -1.29 & 0.18 & -7.23 & .0000 \\
Sign & -0.04 & 0.00 & -0.43 & 0.05 & -9.12 & .0000 \\
ABS(curv)_NormI & -0.02 & 0.01 & -0.02 & 0.00 & -3.78 & .0002 \\
\hline
\end{tabular}

Note-See text for further details. 
The present large-scale study was designed to provide solid empirical data to answer the question formulated above. We presented 260 contour stimuli, derived from line drawings of everyday objects (Snodgrass \& Vanderwart, 1980) to 161 observers and asked them to mark salient points along the contours. Each stimulus was marked by about 40 subjects and our database contains over 200,000 marked points. We have analyzed this database in several ways to test Attneave's (1954) hypothesis, and we have attempted to find stimulus factors that correlate with the empirically obtained saliency values.

Regarding Attneave's (1954) hypothesis, the results from all of the analyses clearly converged in the conclusion that curvature extrema are reported by participants to be more salient than are other points, such as inflections. More than $85 \%$ of the marked points were closer to a local curvature maximum $(M+)$ or negative minimum $(m-)$ than to an inflection $(I)$. Points that were marked more than once were predominantly closer to $M+$ or to $m$ - than to $I$. High values of smoothed saliency occurred almost exclusively at points that are closest to extrema. Very salient points were also very close to either $M+$ or $m-$ (4 pixels or less). When the empirically obtained saliency peaks are plotted on the contours, along with the mathematically computed curvature singularities, the results are quite convincing (see Figure 6): Strong curvature extrema appear to be highly salient points. However, some salient points do not occur at high peaks of positive or negative curvature. Clearly, more than just local absolute curvature determines perceptual saliency of points along the contour. Likewise, for equal absolute curvature values, negative ones were not more salient than positive ones (rather the opposite), showing that our subjects' markings of salient points are determined by other factors than mere information value as derived from a mathematical and local analysis (Feldman \& Singh, 2005).

In an extensive correlational analysis, we have shown that the strongest factor underlying perceptual saliency is the turning angle between the line segments connecting a salient point to each of its two nearest neighboring salient points: A contour point is more salient when its neighboring line segments form a sharp angle. This result converges quite nicely with neurophysiological evidence showing that cells in monkey area V4 are more tuned to specific angles between the two line segments of a corner than to the degree of curvature at the apex of the angle (Pasupathy \& Connor, 1999, 2001). Moreover, two important factors determining point saliency were more global characteristics of the part defined by a triplet of salient points (a corner point and its two nearest neighbors), stick out (Hoffman \& Singh, 1997) and compactness (Zusne, 1970). A point is more salient when the 2-D part surrounding it is less compact and sticks out more.

It is worth pointing out the differences between our study and the earlier ones by Attneave (1954) and Norman et al. (2001) in terms of stimuli (outlines derived from line drawings of everyday objects vs. random shapes), instructions (selecting and marking a freely chosen number of "salient" points along the contour vs. ten points selected for reconstruction) and procedures (quickly viewing and marking the outlines vs. first marking dots next to the shape and then meticulously copying them onto the contour). Yet despite these considerable differences, the results were quite comparable, which is reassuring with respect to the methodological validity of both approaches. Our method allowed us to test many more shapes and subjects. This strengthened the external validity of our results, and enabled the extensive correlational analysis of factors determining perceptual saliency of points along the contour of shapes.

Moreover, the tools that we have developed to analyze these data (e.g., smoothing and thresholding the raw frequency data) can be used to select salient points for other studies using different experimental paradigms to test the role of particular contour points for recognition more directly. Indeed, our methods, like those used by Attneave (1954) and Norman et al. (2001), were not used to investigate the role of curvature extrema for shape recognition. Although Attneave and Norman et al. asked subjects to select points for shape reconstruction, they did not actually test whether the shapes that could be reconstructed from these selected points would then be recognizable by an independent sample of subjects. We have actually done this since performing the present study. In one study, we have created straight-line versions derived from the contour stimuli (as in the case of Attneave's cat), and we have confirmed that they are more identifiable when the straight-line segments connect curvature extrema than when they connect inflections, and also when they connect salient points (using the data from the present study) rather than points midway between salient points (see De Winter \& Wagemans, in press). In another study, we placed contour fragments on the selected points (see Kennedy \& Domander, 1985) and now fragmented versions were generally more identifiable when the fragments were placed on midpoints rather than on salient points (see Panis et al., in press, Experiments 1 and 2). When only a restricted number of dots were shown, outlines were more identifiable with dots on salient points than on midpoints (see Panis et al., in press, Experiment 3). Furthermore, in both of these studies, the variability between stimuli was considerable (see also Panis \& Wagemans, 2007). These differences - between stimuli and between different ways of testing identifiability of outlines reconstructed from salient points-support the need to study the role of curvature singularities thoroughly, using a variety of paradigms. We consider the present study an important step in such an extensive investigation.

In sum, our study has clearly confirmed Attneave's (1954) hypothesis that curvature extrema are salient points, while also showing that the perceptual saliency of a point is determined strongly by nonlocal factors - such as the turning angle between two neighboring line segments and how far the 2-D part sticks out from the shape. This combination of local, semilocal, and global influences on shape perception appears as a recurring theme in this line of work (see also De Winter \& Wagemans, 2004, 2006; Panis et al., in press; Panis \& Wagemans, 2007; Vandekerckhove, Panis, \& Wagemans, 2007). Moreover, our study has provided an extensive benchmark data set to test future ideas about the perceptual saliency of contour 
points or even about shape perception in general (as long as contour curvature plays a role).

\section{AUTHOR NOTE}

This research was supported by a research grant from the University Research Council (OT/00/007) and from the Fund for Scientific Research (FWO-Vlaanderen G.0189.02) to J.W. We thank Sven Panis for assistance with the data collection and for interesting discussions, and Jim Enns, John Kennedy, and two anonymous reviewers for helpful comments on an earlier version of this article. After the first submission of the manuscript, Joeri De Winter died in a tragic accident. Correspondence concerning this article should be addressed to J. Wagemans, Laboratory of Experimental Psychology, University of Leuven, Tiensestraat 102, B-3000, Leuven, Belgium (e-mail: johan.wagemans@psy.kuleuven.be).

\section{REFERENCES}

Attneave, F. (1954). Some informational aspects of visual perception. Psychological Review, 61, 183-193.

De Winter, J., \& Wagemans, J. (2004). Contour-based object identification and segmentation: Stimuli, norms and data, and software tools. $B e-$ havior Research Methods, Instruments, \& Computers, 36, 604-624.

De Winter, J., \& Wagemans, J. (2006). Segmentation of object outlines into parts: A large-scale integrative study. Cognition, 99, 275-325.

De Winter, J., \& Wagemans, J. (in press). The awakening of Attneave's sleeping cat: Identification of everyday objects on the basis of straightline versions of outlines. Perception.

Feldman, J., \& Singh, M. (2005). Information along contours and object boundaries. Psychological Review, 112, 243-252.

Hoffman, D. D., \& Richards, W. A. (1984). Parts of recognition. Cognition, 18, 65-96.

Hoffman, D. D., \& Singh, M. (1997). Salience of visual parts. Cognition, 63, 29-78.

Kennedy, J. M., \& Domander, R. (1985). Shape and contour: The points of maximum change are least useful for recognition. Perception, 14, 367-370

Kennedy, J. M., Juricevic, I., \& BaI, J. (2003). Line and borders of surfaces: Grouping and foreshortening. In H. Hecht, R. Schwartz, \& M. Atherton (Eds.), Looking into pictures: An interdisciplinary approach to pictorial space (pp. 321-354). Cambridge, MA: MIT Press.

KoENDERINK, J. J. (1984). What does the occluding contour tell us about solid shape? Perception, 13, 321-330.

KoEnderink, J. J., \& VAN DoORn, A. J. (1976). The singularities of the visual mapping. Biological Cybernetics, 24, 51-59.
Koenderink, J. J., \& VAn Doorn, A. J. (1982). The shape of smooth objects and the way contours end. Perception, 11, 129-137.

Norman, J. F., Phillips, F., \& Ross, H. E. (2001). Information concentration along the boundary contours of naturally shaped solid objects. Perception, 30, 1285-1294.

Op DE BeECK, H., Wagemans, J., \& Vogels, R. (2001). Inferotemporal neurons represent low-dimensional configurations of parameterized shapes. Nature Neuroscience, 4, 1244-1252.

Op de Beeck, H., Wagemans, J., \& Vogels, R. (2003). Asymmetries in stimulus comparisons by monkey and man. Current Biology, 13, 1803-1808.

Panis, S., De Winter, J., Vandekerckhove, J., \& Wagemans, J. (in press). Identification of everyday objects on the basis of fragmented versions of outlines. Perception.

Panis, S., \& Wagemans, J. (2007). Time-course contingencies in perceptual organization and identification of fragmented object outlines. Manuscript submitted for publication.

Pasupathy, A., \& ConNor, C. E. (1999). Responses to contour features in macaque area V4. Journal of Neurophysiology, 82, 2490-2502.

Pasupathy, A., \& ConNor, C. E. (2001). Shape representation in area V4: Position-specific tuning for boundary conformation. Journal of Neurophysiology, 86, 2505-2519.

Pasupathy, A., \& Connor, C. E. (2002). Population coding of shape in area V4. Nature Neuroscience, 5, 1332-1338.

RESNIKOFF, H. L. (1989). The illusion of reality. New York: Springer.

SABRA, A. I. (1989). The optics of Ibn al-Haytham. London: Warburg Institute.

SnOdgrass, J. G., \& VANDERWART, M. (1980). A standardized set of 260 pictures: Norms for name agreement, image agreement, familiarity, and visual complexity. Journal of Experimental Psychology: Human Learning \& Memory, 6, 174-215.

Vandekerckhove, J., Panis, S., \& Wagemans, J. (2007). The concavity effect is a compound of local and global effects. Perception \& Psychophysics, 69, 1253-1260.

Van Gool, L. J., Moons, T., Pauwels, E., \& Wagemans, J. (1994). Invariance from the Euclidean geometer's perspective. Perception, 23, 547-561.

Wagemans, J., De Winter, J., Op De Beeck, H., Ploeger, A., BeckERS, T., \& VANROOSE, P. (in press). Identification of everyday objects on the basis of silhouette and outline versions. Perception.

Wagemans, J., Notebaert, W., \& Boucart, M. (1998). Lorazepam but not diazepam impairs identification of pictures on the basis of specific contour fragments. Psychopharmacology, 138, 326-333.

Zusne, L. (1970). Visual perception of form. New York: Academic Press. 


\section{APPENDIX}

As explained in the Method section, the stimuli used in this study are 260 outlines of everyday objects derived from the original set of line drawings by Snodgrass and Vanderwart (1980). In another study with 183 different subjects (Wagemans et al., 2007), we have obtained identification norms for these outline versions. On our Web site we make all of the stimuli and norms available, as well as some additional information like the number of inflections along the contours (ppw.kuleuven.be/labexppsy/johanw/index.htm). Here we only report how the 260 stimuli were divided into 4 balanced subsets of 65 stimuli (the numbers refer to those in Snodgrass \& Vanderwart, 1980):

\section{Subset A}

Stimuli 2, 6, 14, 17, 19, 20, 26, 27, 28, 32, 39, 50, 52, 57, 58, 65, 69, 72, 74, 80, 83, 85, 87, 98, 100, 110, 111, $118,120,123,131,137,142,143,147,149,157,158,159,160,165,167,174,176,177,188,193,194,200$, 205, 208, 209, 216, 220, 226, 228, 230, 232, 235, 236, 246, 250, 256, 259, and 260.

\section{Subset B}

Stimuli 1, 4, 7, 10, 11, 18, 24, 25, 38, 40, 43, 48, 53, 54, 60, 62, 66, 68, 78, 81, 88, 89, 95, 96, 97, 99, 105, $108,109,116,117,124,126,127,130,138,148,150,151,153,154,155,162,163,170,173,181,183,184$, $191,192,201,204,212,214,223,224,227,229,233,237,238,242,257$, and 258.

\section{Subset C}

Stimuli $8,12,15,35,37,45,47,49,56,61,71,76,77,79,82,90,91,92,93,94,102,104,106,107,112$, $119,121,125,128,129,133,134,135,139,140,141,145,152,161,164,166,180,182,187,189,195,196$, 198, 199, 202, 206, 207, 210, 211, 213, 217, 219, 222, 225, 239, 243, 244, 247, 253, and 255.

\section{Subset D}

Stimuli 3, 5, 9, 13, 16, 21, 22, 23, 29, 30, 31, 33, 34, 36, 41, 42, 44, 46, 51, 55, 59, 63, 64, 67, 70, 73, 75, 84, $86,101,103,113,114,115,122,132,136,144,146,156,168,169,171,172,175,178,179,185,186,190$, $197,203,215,218,221,231,234,240,241,245,248,249,251,252$, and 254.

As can be seen in the table below, these four subsets were comparable in number of inflections (mean and $S D$ for each subset of 65 stimuli), the average identifiability (in percentages, with $S D$ s), and the number of living objects within each subset of 65 stimuli.

\begin{tabular}{|c|c|c|c|c|c|}
\hline \multirow[b]{2}{*}{ Subset } & \multicolumn{2}{|c|}{$\begin{array}{l}\text { Number of } \\
\text { Inflections }\end{array}$} & \multicolumn{2}{|c|}{ Identifiability } & \multirow{2}{*}{$\begin{array}{c}\text { Number of } \\
\text { Living }\end{array}$} \\
\hline & $M$ & $S D$ & $M$ & $S D$ & \\
\hline A & 16.5 & 15.1 & 63.7 & 37.3 & 16 \\
\hline $\mathrm{B}$ & 16.1 & 14.1 & 60.8 & 40.5 & 17 \\
\hline $\mathrm{C}$ & 15.9 & 14.1 & 61.1 & 40.9 & 17 \\
\hline $\mathrm{D}$ & 15.7 & 14.0 & 61.8 & 39.9 & 18 \\
\hline
\end{tabular}

(Manuscript received March 7, 2005;

revision accepted for publication June 22, 2007.) 